\title{
A Novel Optical Coherence Tomography-Based Calcium Scoring System Can Predict Stent Expansion of Moderate and Severe Calcified Lesions
}

\section{Chang Hou}

Department of Cardiology, Peking University People's Hospital

\section{Linjian Yang}

Department of Cardiology, Peking University People's Hospital

\section{Zixuan Xue}

Department of Cardiology, Peking University People's Hospital Haimiao Lin

Department of Cardiology, Peking University People's Hospital

\section{Yuliang Ma}

Department of Cardiology, Peking University People's Hospital

Qi Li

Department of Cardiology, Peking University People's Hospital

\section{Chuanfen Liu}

Department of Cardiology, Peking University People's Hospital

\section{Mingyu Lu}

Department of Cardiology, Peking University People's Hospital

\section{Hong Zhao}

Department of Cardiology, Peking University People's Hospital

Jian Liu ( $\nabla$ driianliu@163.com )

Department of Cardiology, Peking University People's Hospital

\section{Research Article}

Keywords: calcified plaque, optical coherence tomography, stent underexpansion, percutaneous coronary intervention, calcium score

Posted Date: February 15th, 2022

DOI: https://doi.org/10.21203/rs.3.rs-1343260/v1 
License: (c) (i) This work is licensed under a Creative Commons Attribution 4.0 International License. Read Full License 


\section{Abstract}

Background: Coronary calcified lesions have a serious effect on stent expansion. Researchers have developed a calcium scoring system based on optical coherence tomography (OCT) to identify relatively mild calcified lesions that would benefit from plaque modification. The present study is designed to establish a novel OCT-based scoring system to predict stent expansion of moderate and severe calcified lesions.

Methods: The present study retrospectively included 33 patients (34 calcified lesions observed on coronary angiography) who underwent OCT-guided percutaneous coronary intervention ( $\mathrm{PCl}$ ) at Peking University People's Hospital from January 2016 to July 2021. We off-line reviewed and analysed the images of coronary angiography and OCT. We then developed a calcium scoring system and found the optimal threshold for the prediction of stent underexpansion in patients with moderate and severe calcified lesions.

Results: The average age of the patients was 67 years. Overall final post-PCl stent expansion was $70.74 \%$, and poor stent expansion (i.e., stent expansion < 80\%) was seen in 23 lesions. Approximately $89 \%$ of the lesions manifested as angiographically visible moderate and severe coronary calcification. The average maximum calcium arc, length and thickness assessed by OCT were $230^{\circ}, 25.10 \mathrm{~mm}$ and $1.18 \mathrm{~mm}$, respectively. Multivariate logistic regression model showed that age (OR: $1.173,95 \% \mathrm{Cl}$ : 1.036 1.438, $\mathrm{p}=0.042$ ) and maximum calcium arc (OR: 1.023, 95\% Cl: 1.008 1.050, $\mathrm{p}=0.021$ ) were independent predictors of stent underexpansion. A novel calcium scoring system was established as: $0.16 \times$ age $+0.03 \times$ maximum calcium arc, and the best cut-off of the system for the prediction of stent underexpansion was 16.87 (sensitivity 0.870 , specificity 0.909 , area under the curve [AUC] $0.925,95 \% \mathrm{Cl}$ : $0.836 \sim 1.014, p<0.001)$. Receiver operating characteristics curve analysis showed that the novel scoring system had larger AUC than the previous scoring system (0.925 vs $0.706, p=0.002)$.

Conclusions: The calcium scoring system based on age and maximum calcium arc obtained from OCT may more accurately predict the risk of poor stent expansion in patients with moderate and severe calcified lesions.

\section{Introduction}

Coronary calcification remains a great challenge of percutaneous coronary intervention (PCl), which has an adverse impact on stent expansion and immediate treatment efficacy (1). The presence of calcified lesions strongly predicts the occurrence of 1-year stent thrombosis and target lesion revascularization (2). It is necessary to perform adequate modification for calcified plaques before stent implantation to achieve excellent expansion.

Optical coherence tomography $(\mathrm{OCT})$ is an emerging intracoronary imaging modality that can accurately identify calcified lesions and assess the severity of calcification (3). Previous study shows that OCTguided PCI for calcified lesions can result in better stent expansion (4). 
Fujino et al have found that calcium lesions with maximum angle $>180^{\circ}$, maximum thickness $>0.5 \mathrm{~mm}$, and length $>5 \mathrm{~mm}$ may be at risk of stent underexpansion in patients with relatively mild calcification (5). However, whether this OCT-based scoring system can be applied to the patients with moderate and severe calcified lesions is unknown. The aim of the present study is to develop a novel scoring system for the prediction of stent underexpansion of moderate and severe calcified lesions.

\section{Methods}

\subsection{Study Population}

78 patients with the existence of calcified lesions on coronary angiography who underwent OCT-guided stent implantation at Peking University People's Hospital (Beijing, China) between January 2016 and July 2021 were enrolled in the present study. All lesions included had an OCT finding of maximum calcium length $>5 \mathrm{~mm}$, thickness $>0.5 \mathrm{~mm}$ or arc $>180^{\circ}$, which were considered as moderate and severe calcified lesions, despite the minority of them showed only mild calcification on coronary angiography. The

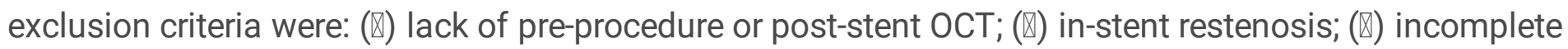
OCT images which were unable to analyze and measure critical parameters and $(\mathbb{\nabla})$ poor image quality.

\subsection{Study Design}

This was a retrospective study to develop a novel OCT-based calcium scoring system to predict stent underexpansion of moderate and severe calcified lesions. We searched and reviewed medical records, coronary angiography and OCT images of the eligible patients. The patient's demographic information, clinical manifestation, past medical history, family history of coronary heart disease, laboratory examinations and $\mathrm{PCl}$ procedural characteristics were recorded.

\subsection{PCI Procedure and OCT Imaging Acquisition}

Coronary angiography and stent implantation were performed according to the standard protocol. OCT data were acquired by frequency-domain OCT $\left(\mathrm{C}^{-\mathrm{XR}^{\mathrm{TM}}}\right.$ or OPTIS $\left.{ }^{\mathrm{TM}}\right)$ and Dragonfly ${ }^{\mathrm{TM}}$ Duo catheter (all Abbott Vascular, Santa Clara, CA, USA). Based on the angiographic and OCT findings, the decision to perform rotational atherectomy (RA) or conventional angioplasty with cutting, scoring or non-compliant balloon prior to stent implantation depended on the operator's discretion and operational skill.

\subsection{Quantitative Coronary Angiography (QCA) Analysis}

All angiography images were analysed with QAngio $\circledast$ XA 7.3 (Medis, Leiden, the Netherlands) software by two independent interventional cardiologists who were blinded to clinical presentation and OCT information of the patients. The degree of calcification was classified according to the Mintz criteria as 
none/mild, moderate, or severe (6). The location, angulation and length of calcified lesions, reference vessel diameter, minimum lumen diameter and diameter stenosis of target lesions were evaluated.

\subsection{OCT Imaging Analysis}

All OCT data were analysed using off-line review workstation (ORW) software (version E.0.2, Abbott Vascular) and dedicated expert consensus reports (7-8) by two independent interventional cardiologists who were blinded to the clinical and angiographic information.

Only lesions with a calcium arc $\geq 30^{\circ}$ were included in the present study. It was considered two separate calcified lesions if there was more than $1 \mathrm{~mm}$ of non-calcified plaque between the lesions. When the boundary of calcified lesions was not obvious, the maximum visible thickness was measured.

It was defined superficial calcification when the distance between the lesion and the lumen was $<100$ $\mu \mathrm{m}$. Stent edge dissection was defined as interruption of surface continuity at the stent edge (i.e., within 5 $\mathrm{mm}$ distal and proximal of the stent). Stent malapposition was defined as the longitudinal distance from the stent surface to the lumen being greater than the stent thickness, whereas tissue protrusion was defined as protrusion of the tissue into the lumen after stent implantation. The percentage of area and diameter stenosis were defined as the minimum lumen area divided by mean reference lumen area, and the mean lumen diameter at narrowest site divided by mean reference lumen diameter, respectively. Stent expansion was calculated as the minimum stent area divided by mean reference vessel area $\times 100 \%$. According to the stent expansion calculated, patients were divided into adequate (i.e., stent expansion $\geq 80 \%$ ) and poor stent expansion groups (8-9).

\subsection{Statistical Analysis}

Statistical analysis was performed using the SPSS software (version 24.0, IBM Corp, NY, USA). Categorical variables were presented as $\mathrm{n}(\%)$ and compared using $\chi^{2}$ or Fisher's exact test, as appropriate. Continuous variables with normal distribution were presented as mean \pm standard deviation, otherwise presented as median and interquartile range, which were compared using the Student's $t$-test or Kruskal-Wallis rank-sum test, as appropriate. Bivariate correlation analysis was performed to identify the variables correlated with stent expansion. Then we investigated the independent predictors of stent underexpansion by including the potential confounding factors in the multivariate logistic regression model with a step-wise algorithm and developed a novel calcium scoring system. Receiver operating characteristics (ROC) curve analysis was used to determine the best cut-off value for the novel scoring system for the prediction of stent underexpansion. The area under the curve (AUC) of the novel scoring system was compared with that proposed by Fujino using $\chi^{2}$ test for two correlated ROC curves. The inter-observer agreements for OCT data were evaluated by calculating the intraclass correlation coefficients (ICC) or $k$ coefficients. A two-sided $p$-value $<0.05$ was considered to indicate statistically significant difference. 


\section{Results}

\subsection{Clinical and Procedural Characteristics}

A total of 53 patients with calcified lesions on coronary angiography were enrolled who underwent stent implantation with the guidance of OCT and had complete pre- or post-stent OCT images. 33 patients (34 lesions) were finally included after excluding the patients with in-stent restenosis and OCT images unable to analyze due to either poor-quality or lacking important frames (Figure 1).

The mean age of the patients was $67 \pm 10$ years, with 20 male. More than $70 \%$ of the patients had the coexisting hypertension and hyperlipidemia. The patients diagnosed with stable angina pectoris were 7 $(21.2 \%)$ whereas acute coronary syndrome were 26 (78.8\%). Poor stent expansion occurred in 22 patients (23 lesions). The age of the patients was significantly older (70 \pm 10 vs $59 \pm 8, p=0.003$ ) and the estimated glomerular filtration rate was significantly lower (82.16 vs $93.95, p=0.036)$ in the stent underexpansion group. There was no significant difference with regard to remaining clinical characteristics between two groups (Table 1).

Table 1. Clinical characteristics of the patients 


\begin{tabular}{|c|c|c|c|}
\hline Variables & $\begin{array}{l}\text { Poor expansion } \\
(\mathrm{n}=22)\end{array}$ & $\begin{array}{l}\text { Adequate expansion } \\
(\mathrm{n}=11)\end{array}$ & P-value \\
\hline Age (year) & $70 \pm 10$ & $59 \pm 8$ & 0.003 \\
\hline Male, n (\%) & $12(54.5 \%)$ & $8(72.7 \%)$ & 0.456 \\
\hline $\mathrm{BMI}\left(\mathrm{kg} / \mathrm{m}^{2}\right)$ & $25.91 \pm 3.80$ & $25.08 \pm 1.58$ & 0.496 \\
\hline Hypertension, n (\%) & $16(72.7 \%)$ & $7(63.6 \%)$ & 0.696 \\
\hline Diabetes, n (\%) & $10(45.5 \%)$ & $4(36.4 \%)$ & 0.719 \\
\hline Hyperlipidemia, n (\%) & $16(72.7 \%)$ & $9(81.8 \%)$ & 0.687 \\
\hline CKD, n (\%) & $2(9.1 \%)$ & $1(9.1 \%)$ & 1.000 \\
\hline Smoking, n (\%) & $8(36.4 \%)$ & $6(54.5 \%)$ & 0.534 \\
\hline Family history of $\mathrm{CHD}, \mathrm{n}(\%)$ & $4(18.2 \%)$ & $5(45.5 \%)$ & 0.121 \\
\hline Prior PCl, n (\%) & $8(36.4 \%)$ & $4(36.4 \%)$ & 1.000 \\
\hline Clinical diagnosis, n (\%) & & & 1.000 \\
\hline Stable angina & $5(22.7 \%)$ & $2(18.2 \%)$ & \\
\hline UA & $13(59.1 \%)$ & $7(63.6 \%)$ & \\
\hline Acute MI & $4(18.2 \%)$ & $2(18.2 \%)$ & \\
\hline LVEF (\%) & $69.03 \pm 5.71$ & $66.32 \pm 6.04$ & 0.298 \\
\hline LDL-C (mmol/L) & $2.06 \pm 0.77$ & $2.06 \pm 0.53$ & 0.992 \\
\hline FPG (mmol/L) & $5.08(4.52,5.58)$ & $5.07(4.72,6.10)$ & 0.620 \\
\hline eGFR $\left(\mathrm{ml} / \mathrm{min} / 1.73 \mathrm{~m}^{2}\right)$ & $82.16(71.37,93.25)$ & $93.95(90.52,104.00)$ & 0.036 \\
\hline
\end{tabular}

Abbreviations: $\mathrm{BMI}$, body mass index; $\mathrm{CKD}$, chronic kidney disease; $\mathrm{CHD}$, coronary heart disease; $\mathrm{PCl}$, percutaneous coronary intervention; $\mathrm{UA}$, unstable angina; $\mathrm{MI}$, myocardial infarction; LVEF, left ventricular ejection fraction; LDL-C, low density lipoprotein-cholesterol; FPG, fasting plasma glucose; eGFR, estimated glomerular filtration rate.

Values are presented as number (\%), mean \pm standard deviation, or median (interquartile range).

RA was used in the modification of 19 (55.9\%) lesions. The lesions treated with scoring or non-compliant balloon prior to stent deployment were both $13(38.2 \%)$. The average amount of the stent implanted was 2 and the total length of stents was $44 \mathrm{~mm}$. Compared with the patients in the adequate stent expansion group, the rate of RA performed during $\mathrm{PCl}$ was significantly higher in the patients with stent underexpansion (73.9\% vs $18.2 \%, p=0.003$ ) (Table 2 ). 
Table 2. Procedural characteristics of the lesions

\begin{tabular}{|llll|}
\hline Variables & $\begin{array}{l}\text { Poor expansion } \\
(\mathrm{n}=23)\end{array}$ & $\begin{array}{l}\text { Adequate expansion } \\
(\mathrm{n}=11)\end{array}$ & P-value \\
\hline Scoring balloon, $\mathrm{n}(\%)$ & $11(47.8 \%)$ & $2(18.2 \%)$ & 0.245 \\
\hline Maximum pressure $(\mathrm{atm})$ & $14.00(11.00,15.00)$ & $12.00(12.00,12.00)$ & 0.545 \\
\hline Maximum diameter $(\mathrm{mm})$ & $2.75(2.50,2.88)$ & $2.50(2.38,2.62)$ & 0.351 \\
\hline Non-compliant balloon, $\mathrm{n}(\%)$ & $11(47.8 \%)$ & $2(18.2 \%)$ & 0.245 \\
\hline Maximum pressure $(\mathrm{atm})$ & $18.00(17.00,24.00)$ & $13.50(12.75,14.25)$ & 0.091 \\
\hline Maximum diameter $(\mathrm{mm})$ & $2.50(2.50,2.50)$ & $2.25(2.12,2.38)$ & 0.098 \\
\hline Semi-compliant balloon, $\mathrm{n}(\%)$ & $18(78.3 \%)$ & $8(72.7 \%)$ & 1.000 \\
\hline Maximum pressure $(\mathrm{atm})$ & $16.00(14.00,16.00)$ & $15.00(13.50,16.50)$ & 0.686 \\
\hline Maximum diameter $(\mathrm{mm})$ & $2.50(2.12,2.50)$ & $2.50(2.38,2.50)$ & 0.885 \\
\hline RA, $\mathrm{n}(\%)$ & $17(73.9 \%)$ & $2(18.2 \%)$ & 0.003 \\
\hline Times of RA & $5(3,7)$ & $5(4,5)$ & 0.893 \\
\hline Maximum burr size $(\mathrm{mm})$ & $1.50(1.38,1.50)$ & $1.50(1.50,1.50)$ & 0.725 \\
\hline Speed of burr $(\mathrm{r} / \mathrm{min})$ & $15.00(15.00,16.00)$ & $16.60(15.90,17.30)$ & 0.212 \\
\hline Stent & & & $1(1,2)$ \\
\hline Number of stent & $2(1,3)$ & $32.00(28.00,57.00)$ & 0.197 \\
\hline Total stent length $(\mathrm{mm})$ & $48.00(37.50,69.00)$ & 0.165 \\
\hline Maximum diameter $(\mathrm{mm})$ & $3.00(2.75,3.00)$ & $2.75(2.50,3.00)$ & 0.126 \\
\hline
\end{tabular}

Abbreviations: RA, rotational atherectomy.

Values are presented as number (\%), or median (interquartile range).

\subsection{Imaging Analysis of Calcified Plaques}

The majority of patients had multi-vessel disease. The target lesions were mainly located in the left anterior descending artery. The prevalence of moderate and severe coronary calcification assessed by angiography was up to $88.2 \%$ (30 lesions). All parameters of QCA analysis were comparable between two groups (Table 3).

Table 3. QCA analyses of calcified lesions 


\begin{tabular}{|c|c|c|c|}
\hline Variables & $\begin{array}{l}\text { Poor expansion } \\
(n=23)\end{array}$ & $\begin{array}{l}\text { Adequate expansion } \\
(\mathrm{n}=11)\end{array}$ & P-value \\
\hline \multicolumn{4}{|l|}{ Pre-procedure } \\
\hline Multivessel disease, n (\%) & $19(82.6 \%)$ & $11(100.0 \%)$ & 0.280 \\
\hline Target vessel, n (\%) & & & 1.000 \\
\hline Left anterior descending & $19(82.6 \%)$ & $9(81.8 \%)$ & \\
\hline Left circumflex & $1(4.3 \%)$ & $0(0.0 \%)$ & \\
\hline Right coronary artery & $3(13.0 \%)$ & $2(18.2 \%)$ & \\
\hline Degree of calcification, n (\%) & & & 0.362 \\
\hline None or mild & $2(8.7 \%)$ & $2(18.2 \%)$ & \\
\hline Moderate & $14(60.9 \%)$ & $8(72.7 \%)$ & \\
\hline Severe & $7(30.4 \%)$ & $1(9.1 \%)$ & \\
\hline Bifurcation, n (\%) & $2(8.7 \%)$ & $4(36.4 \%)$ & 0.070 \\
\hline Angulation, n (\%) & & & 0.203 \\
\hline$<90^{\circ}$ & $22(95.7 \%)$ & $10(90.9 \%)$ & \\
\hline$>90^{\circ}$ & $1(4.3 \%)$ & $1(9.1 \%)$ & \\
\hline Calcium length (mm) & $35.77 \pm 20.66$ & $26.03 \pm 12.34$ & 0.227 \\
\hline RVD (mm) & $2.63(2.38,2.83)$ & $2.54(2.32,2.64)$ & 0.597 \\
\hline MLD (mm) & $1.24 \pm 0.42$ & $1.33 \pm 0.33$ & 0.579 \\
\hline Diameter stenosis (\%) & $53.43 \pm 13.27$ & $48.02 \pm 12.92$ & 0.290 \\
\hline \multicolumn{4}{|l|}{ Post-procedure } \\
\hline RVD (mm) & $2.39(2.12,2.53)$ & $2.50(2.26,2.94)$ & 0.155 \\
\hline MSD (mm) & $2.19(2.02,2.44)$ & $2.33(2.02,2.74)$ & 0.382 \\
\hline Diameter stenosis (\%) & $11.58(6.45,15.02)$ & $10.71(6.80,15.51)$ & 0.887 \\
\hline
\end{tabular}

Abbreviations: QCA, quantitative coronary angiography; RVD, reference vessel diameter; MLD, minimum lumen diameter; MSD, minimum stent diameter.

Values are presented as number (\%), mean \pm standard deviation, or median (interquartile range).

There was a good concordance between two observers for the interpretation of OCT images and the assessment of maximum calcium arc $(I C C=0.877)$, thickness $(I C C=0.874)$ and length $(I C C=0.968)$. The lesions all manifested as superficial calcification, with the median maximum calcium arc $230^{\circ}$, median 
maximum calcium length $25.10 \mathrm{~mm}$ and average maximum calcium thickness $1.18 \mathrm{~mm}$, respectively. The overall final post-PCl stent expansion was $70.74 \%$. Notably, the maximum calcium arc $\left(299^{\circ}\right.$ vs $142^{\circ}$, $p<0.001)$ and thickness $(1.24 \mathrm{~mm}$ vs $1.04 \mathrm{~mm}, \mathrm{p}=0.029)$ were larger in the poor stent expansion group, while the pre-stent diameter stenosis obtained from OCT (52.76\% vs $66.29 \%, p=0.038)$ was lower. The proportion of stent malapposition increased significantly in the poor stent expansion group $(60.9 \%$ vs $9.1 \%, p=0.011)($ Table 4).

\section{Table 4. OCT data of calcified lesions}

\begin{tabular}{|llll|}
\hline Variables & $\begin{array}{l}\text { Poor expansion } \\
(\mathrm{n}=23)\end{array}$ & $\begin{array}{l}\text { Adequate expansion } \\
(\mathrm{n}=11)\end{array}$ & P-value \\
\hline Pre-stent & & & \\
\hline Superficial calcium, $\mathrm{n}(\%)$ & $23(100 \%)$ & $11(100 \%)$ & - \\
\hline Maximum calcium length $(\mathrm{mm})$ & $33.15(15.62,40.20)$ & $20.65(14.78,25.10)$ & 0.094 \\
\hline Maximum calcium arc $\left(^{\circ}\right)$ & $299(205,345)$ & $142(104,216)$ & 0.001 \\
\hline Maximum calcium thickness $(\mathrm{mm})$ & $1.24 \pm 0.23$ & $1.04 \pm 0.25$ & 0.029 \\
\hline Minimum lumen area $\left(\mathrm{mm}^{2}\right)$ & $1.64(1.16,2.28)$ & $2.26(1.65,2.71)$ & 0.217 \\
\hline Reference vessel area $\left(\mathrm{mm}^{2}\right)$ & $6.47(5.08,8.20)$ & $5.30(5.11,6.92)$ & 0.429 \\
\hline Diameter stenosis $(\%)$ & $52.76(45.59,58.58)$ & $66.29(55.66,71.74)$ & 0.038 \\
\hline Area stenosis $(\%)$ & $81.73(80.06,83.37)$ & $84.11(82.44,86.80)$ & 0.063 \\
\hline Post-stent & & & 0.063 \\
\hline Tissue prolapse, $\mathrm{n}(\%)$ & $1(4.3 \%)$ & $0(0 \%)$ & 1.000 \\
\hline Stent edge dissection, $\mathrm{n}(\%)$ & $0(0 \%)$ & $0(0 \%)$ & - \\
\hline Stent malapposition, $\mathrm{n}(\%)$ & $13(60.9 \%)$ & $1(9.1 \%)$ & 0.014 \\
\hline Minimum stent area $\left(\mathrm{mm}^{2}\right)$ & $4.25(3.96,5.62)$ & $5.42(4.60,6.77)$ & 0.146 \\
\hline Reference vessel area $\left(\mathrm{mm}^{2}\right)$ & $8.02(6.43,9.09)$ & $5.90(5.33,8.64)$ & 0.217 \\
\hline Stent expansion $(\%)$ & $63.39 \pm 12.72$ & $86.10 \pm 4.59$ & $<01$ \\
\hline
\end{tabular}

Abbreviations: OCT, optical coherence tomography.

Values are presented as number (\%), mean \pm standard deviation, or median (interquartile range).

\subsection{Development of Calcium Scoring System}


The age, utilization of RA, maximum calcium arc, thickness, length and diameter stenosis from OCT significantly correlated with stent expansion. After adjustment of the potential confounding factors, the age (OR: $1.173,95 \% \mathrm{Cl}: 1.036 \sim 1.438, \mathrm{p}=0.042)$ and maximum calcium arc (OR: $1.023,95 \% \mathrm{Cl}$ : 1.008 1.050, $p=0.021$ ) were found to be the independent predictors of stent underexpansion in the multivariate logistic regression model (Table 5). Based on these results, we established a novel calcium scoring system as: $0.16 \times$ age $+0.03 \times$ maximum calcium arc.

\section{Table 5. Multivariate logistic regression model of stent underexpansion}

\begin{tabular}{|lllll|}
\hline Variables & $\beta$-coefficient & Odds Ratio & $95 \% \mathrm{Cl}$ & P-value \\
\hline Age (year) & 0.159 & 1.173 & $1.036 \sim 1.438$ & $\mathbf{0 . 0 4 2}$ \\
\hline Maximum calcium $\operatorname{arc}\left({ }^{\circ}\right)$ & 0.023 & 1.023 & $1.008 \sim 1.050$ & $\mathbf{0 . 0 2 1}$ \\
\hline
\end{tabular}

Abbreviations: $\mathrm{Cl}$, confidence interval.

We then identified the optimal threshold for the prediction of stent underexpansion by ROC curve analysis. The best cut-off value for the scoring system was 16.87 (sensitivity 0.870 , specificity 0.909 , AUC 0.925, 95\% Cl: 0.836 1.014, p < 0.001) (Figure 2). Representative OCT images were shown in Figure 3.

\subsection{Comparison with Previous Scoring System}

Compared with the calcium scoring system proposed by Fujino, the novel system according to the age and maximum calcium arc had better performance for the prediction of stent underexpansion in the present study population, with a significantly larger AUC of ROC (0.925 vs $0.706, p=0.002)$ (Figure 2).

\section{Discussion}

The present study establishes a novel system to effectively predict the stent expansion of moderate and severe calcified lesions by retrospectively analyzing the procedural characteristics and intracoronary imaging data of the patients with moderate and severe coronary calcification who undergo PCl. The main

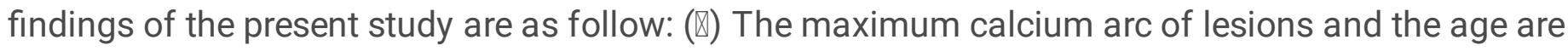
independent predictors of stent underexpansion in patients with moderate and severe coronary calcification. ( () The calcium scoring system based on these parameters can accurately predict the risk of stent underexpansion and guide the strategy of lesion modification such as RA in patients with moderate and severe coronary calcification.

It has been shown that the occurrence of stent underexpansion is significantly increased in patients with severe calcified lesions and incomplete stent expansion is known to be the common risk factor of stent thrombosis and in-stent restenosis (10-12). Severe coronary calcification is also an independent predictor of worse prognosis and increases the rate of mortality and other adverse cardiac events (13-14). 
Therefore, more aggressive strategy of lesion modification should be used before stent deployment to achieve optimal interventional treatment effect (15). Matsuhiro et al find the maximum calcium thickness $<880 \mu \mathrm{m}$ is a useful predictor of acceptable stent expansion (defined by $80 \%$ ) in moderate calcified lesions (16). In another study Maejima et al conclude that larger calcium arc and thinner calcium thickness are associated with formation of calcium crack, which is the important determinant of optimal stent expansion (17). However, in the present study we find the maximum calcium arc not thickness of lesions and the age of patients have a significant impact on stent expansion. Furthermore, we propose a new calcium scoring system based on these parameters which shows a high accuracy in predicting the risk of stent underexpansion. The proportion of RA during $\mathrm{PCl}$ is significantly higher in patients with poor stent expansion, possibly because the calcification of lesions is more severe in this group.

OCT has greater capability than coronary angiography in the detection of calcium with a sensitivity ranging from $95 \%$ to $96 \%$ and specificity of $97 \%$, respectively (18-19). In addition, compared with visual angiographic assessment alone, intracoronary OCT images can provide more information and parameters regarding the calcification severity of target lesions, such as maximum calcium arc, thickness, depth and longitudinal length. In the present study, the maximum calcium arc obtained from OCT images is proven to be an independent predictor of stent expansion, while parameters from QCA not. This finding highlights the importance and great value of intravascular imaging modality in the interventional strategy-making process of severe calcified lesions.

In the previous study, it's reported that for lesions with none/mild calcification the rate of major adverse cardiac events at 1 year is only $8.3 \%$, whereas $14.6 \%$ and $17.7 \%$ for moderate and severe calcified lesions, respectively (20). Fujino et al develop an OCT-based calcium scoring system to identify lesions which may be at risk of stent underexpansion and benefit from plaque modification prior to stent implantation. It's worthy to note that only $29.7 \%$ of the patients enrolled have moderate and severe calcified lesions assessed by angiography and OCT characteristics of the patients in this study orient towards relatively mild calcification. Patients treated with RA or scoring balloon are excluded (5). In contrast, up to $88.2 \%$ of the lesions have angiographically visible moderate and severe calcification in the present study. We also compare these two systems and confirm that the newly-established system has better performance of predicting the immediate therapeutic outcome of $\mathrm{PCl}$ than the widely used scoring system by Fujino in patients with moderate and severe coronary calcification.

The present study has several limitations. First, the study is retrospective with a relatively small sample size. Hence, the conclusion drawn from the present study may be underpower. Second, there is lack of a specific cohort to validate the accuracy of the calcium scoring system to predict stent underexpansion. Third, since the OCT images are not read and analysed by the same interventional cardiologist at different time, the intra-observer concordance for the assessment of OCT data cannot be obtained. Next, the information regarding peri-procedural complications such as coronary dissection or perforation and the balloon used for post-dilatation is not available. Finally, the capability of the calcium scoring system to predict the long-term clinical outcome post $\mathrm{PCl}$ in patients with moderate and severe calcified lesions is unknown. 


\section{Conclusions}

The novel OCT-based calcium scoring system shows a high accuracy for the prediction of the occurrence of stent underexpansion in patients with moderate and severe coronary calcification, which needs further validation in a larger cohort.

\section{Abbreviations}

OCT: Optical coherence tomography; PCl: Percutaneous coronary intervention; RA: Rotational atherectomy; ORW: Off-line review workstation; ROC: Receiver operating characteristics; AUC: Area under the curve; ICC: Intraclass correlation coefficients.

\section{Declarations}

\section{Ethics approval and consent to participate}

This study was approved by the Ethics Committee of Peking University People's Hospital and conducted according to the principles of the Declaration of Helsinki. Since this clinical study was a retrospective analysis of the information of previous cases, without direct contact with the subjects and subject privacy protection, the risk borne by the subjects was not greater than the minimum risk. The Ethics Committee of Peking University People's Hospital agreed to exempt informed consent after review.

\section{Consent for publication}

Not applicable.

\section{Availability of data and materials' statement}

The datasets used and/or analysed during the current study are available from the corresponding author on reasonable request.

\section{Competing interests}

The authors declare that they have no competing interests.

\section{Funding}

This study was supported by the National Natural Science Foundation of China (No.11832003, No.81970294), and Peking University People's Hospital Scientific Research Development Funds (RDL2020-11).

\section{Authors' contributions}


$\mathrm{CH}$ and $\mathrm{LY}$ collected, analyzed and interpreted the patient data, and were major contributors to writing the manuscript. ZX and HL helped to collect and analyze the data. YM, QL, ML, HZ and JL performed the operation. $\mathrm{CL}$ revised the manuscript. JL designed the present study. All authors have read and approved the final version of this manuscript.

\section{Acknowledgements}

The authors sincerely thank Zhuang Tao, PhD, for his help in the present study.

\section{Authors' information}

Dr. Chang Hou, Email: hc199051@163.com

Dr. Linjian Yang, Email: yanglj9973@yeah.net

Dr. Zixuan Xue, Email: 1260020763@qq.com

Dr. Haimiao Lin, Email: 1710301238@pku.edu.cn

Dr. Yuliang Ma, Email: newmourice@163.com

Dr. Qi Li, Email: sdlq5726@sina.com

Dr. Chuanfen Liu, Email: liucf-99@163.com

Dr. Mingyu Lu, Email: abc2625@sina.com.cn

Dr. Hong Zhao, Email: hong.zhao@medmail.com.cn

Dr. Jian Liu, Email: drjianliu@163.com

\section{References}

1. Kobayashi Y, Okura H, Kume T, Yamada R, Kobayashi Y, Fukuhara K, et al. Impact of target lesion coronary calcification on stent expansion. Circulation journal : official journal of the Japanese Circulation Society. 2014;78(9):2209-14.

2. Généreux P, Madhavan MV, Mintz GS, Maehara A, Palmerini T, Lasalle L, et al. Ischemic outcomes after coronary intervention of calcified vessels in acute coronary syndromes. Pooled analysis from the HORIZONS-AMI (Harmonizing Outcomes With Revascularization and Stents in Acute Myocardial Infarction) and ACUITY (Acute Catheterization and Urgent Intervention Triage Strategy) TRIALS. Journal of the American College of Cardiology. 2014;63(18):1845-54.

3. Mehanna E, Bezerra HG, Prabhu D, Brandt E, Chamié D, Yamamoto H, et al. Volumetric characterization of human coronary calcification by frequency-domain optical coherence 
tomography. Circulation journal : official journal of the Japanese Circulation Society. 2013;77(9):2334-40.

4. Kobayashi $N$, Ito $Y$, Yamawaki M, Araki M, Obokata M, Sakamoto $Y$, et al. Optical coherence tomography-guided versus intravascular ultrasound-guided rotational atherectomy in patients with calcified coronary lesions. Eurolntervention : journal of EuroPCR in collaboration with the Working Group on Interventional Cardiology of the European Society of Cardiology. 2020;16(4):e313-e21.

5. Fujino A, Mintz GS, Matsumura M, Lee T, Kim SY, Hoshino M, et al. A new optical coherence tomography-based calcium scoring system to predict stent underexpansion. Eurolntervention : journal of EuroPCR in collaboration with the Working Group on Interventional Cardiology of the European Society of Cardiology. 2018;13(18):e2182-e9.

6. Mintz GS, Popma JJ, Pichard AD, Kent KM, Satler LF, Chuang YC, et al. Patterns of calcification in coronary artery disease. A statistical analysis of intravascular ultrasound and coronary angiography in 1155 lesions. Circulation. 1995;91(7):1959-65.

7. Prati F, Guagliumi G, Mintz GS, Costa M, Regar E, Akasaka T, et al. Expert review document part 2: methodology, terminology and clinical applications of optical coherence tomography for the assessment of interventional procedures. European heart journal. 2012;33(20):2513-20.

8. Tearney GJ, Regar E, Akasaka T, Adriaenssens T, Barlis P, Bezerra HG, et al. Consensus standards for acquisition, measurement, and reporting of intravascular optical coherence tomography studies: a report from the International Working Group for Intravascular Optical Coherence Tomography Standardization and Validation. Journal of the American College of Cardiology. 2012;59(12):105872.

9. Räber L, Mintz GS, Koskinas KC, Johnson TW, Holm NR, Onuma Y, et al. Clinical use of intracoronary imaging. Part 1: guidance and optimization of coronary interventions. An expert consensus document of the European Association of Percutaneous Cardiovascular Interventions. European heart journal. 2018;39(35):3281-300.

10. Mosseri M, Satler LF, Pichard AD, Waksman R. Impact of vessel calcification on outcomes after coronary stenting. Cardiovascular revascularization medicine : including molecular interventions. 2005;6(4):147-53.

11. Fujii K, Carlier SG, Mintz GS, Yang YM, Moussa I, Weisz G, et al. Stent underexpansion and residual reference segment stenosis are related to stent thrombosis after sirolimus-eluting stent implantation: an intravascular ultrasound study. Journal of the American College of Cardiology. 2005;45(7):995-8.

12. Kastrati A, Dibra A, Mehilli J, Mayer S, Pinieck S, Pache J, et al. Predictive factors of restenosis after coronary implantation of sirolimus- or paclitaxel-eluting stents. Circulation. 2006;113(19):2293-300.

13. Bourantas CV, Zhang YJ, Garg S, Iqbal J, Valgimigli M, Windecker S, et al. Prognostic implications of coronary calcification in patients with obstructive coronary artery disease treated by percutaneous coronary intervention: a patient-level pooled analysis of 7 contemporary stent trials. Heart (British Cardiac Society). 2014;100(15):1158-64. 
14. Sharma SK, Bolduan RW, Patel MR, Martinsen BJ, Azemi T, Giugliano G, et al. Impact of calcification on percutaneous coronary intervention: MACE-Trial 1-year results. Catheterization and cardiovascular interventions : official journal of the Society for Cardiac Angiography \& Interventions. 2019;94(2):18794.

15. Tang Z, Bai J, Su SP, Lee PW, Peng L, Zhang T, et al. Aggressive plaque modification with rotational atherectomy and cutting balloon for optimal stent expansion in calcified lesions. Journal of geriatric cardiology : JGC. 2016;13(12):984-91.

16. Matsuhiro Y, Nakamura D, Shutta R, Yanagawa K, Nakamura H, Okamoto N, et al. Maximum calcium thickness is a useful predictor for acceptable stent expansion in moderate calcified lesions. The international journal of cardiovascular imaging. 2020;36(9):1609-15.

17. Maejima N, Hibi K, Saka K, Akiyama E, Konishi M, Endo M, et al. Relationship Between Thickness of Calcium on Optical Coherence Tomography and Crack Formation After Balloon Dilatation in Calcified Plaque Requiring Rotational Atherectomy. Circulation journal : official journal of the Japanese Circulation Society. 2016;80(6):1413-9.

18. Yabushita H, Bouma BE, Houser SL, Aretz HT, Jang IK, Schlendorf KH, et al. Characterization of human atherosclerosis by optical coherence tomography. Circulation. 2002;106(13):1640-5.

19. Wang X, Matsumura M, Mintz GS, Lee T, Zhang W, Cao Y, et al. In Vivo Calcium Detection by Comparing Optical Coherence Tomography, Intravascular Ultrasound, and Angiography. JACC Cardiovascular imaging. 2017;10(8):869-79.

20. Copeland-Halperin RS, Baber U, Aquino M, Rajamanickam A, Roy S, Hasan C, et al. Prevalence, correlates, and impact of coronary calcification on adverse events following $\mathrm{PCl}$ with newergeneration DES: Findings from a large multiethnic registry. Catheterization and cardiovascular interventions : official journal of the Society for Cardiac Angiography \& Interventions. 2018;91(5):85966.

\section{Figures}




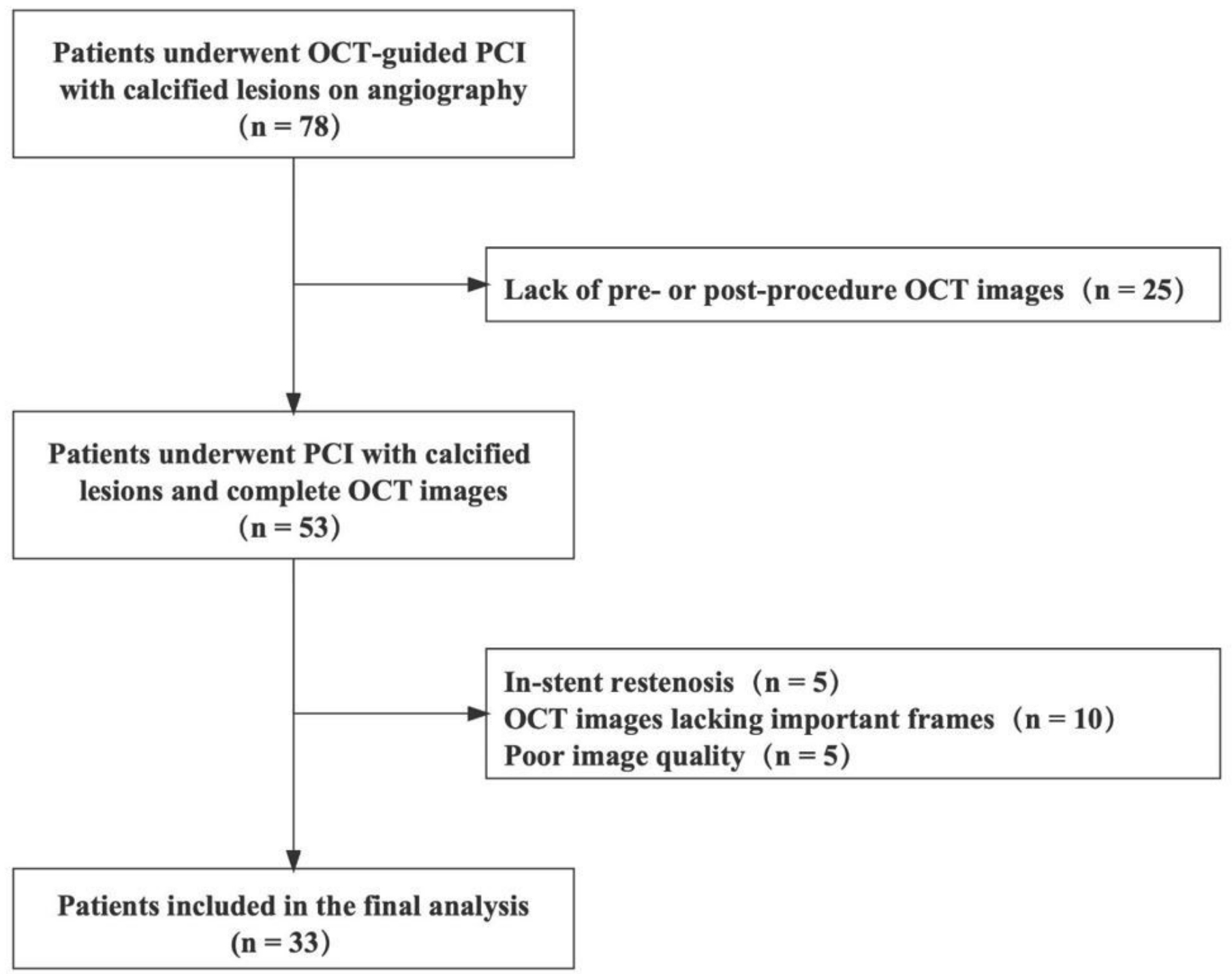

Figure 1

Study flowchart. 


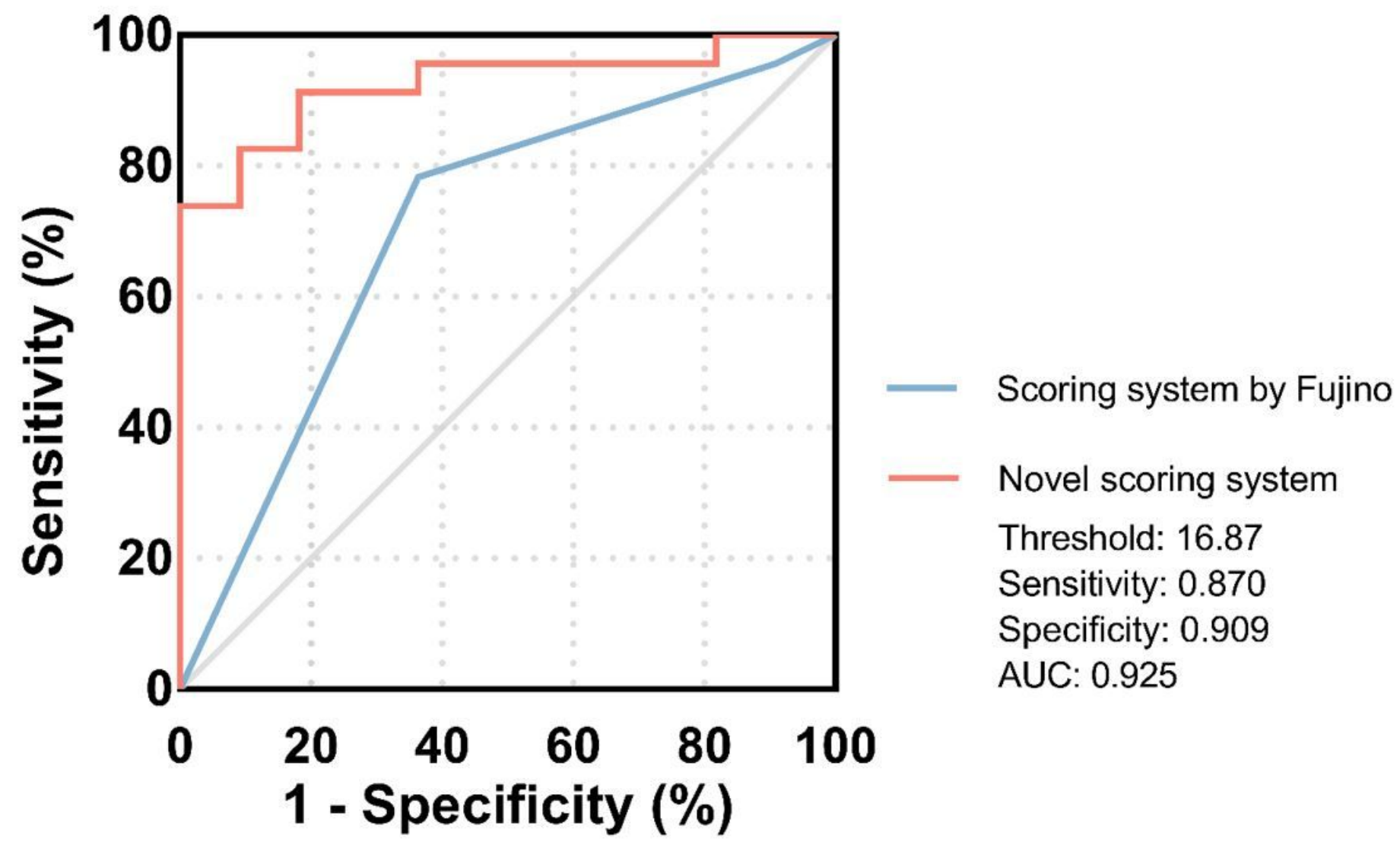

Figure 2

Receiver operating characteristics curves of novel calcium scoring system and that proposed by Fujino. The optimal threshold of novel calcium scoring system for predicting stent underexpansion is 16.87 with sensitivity 0.870 , specificity 0.909 and area under the curve (AUC) 0.925 , respectively $(p<0.001)$. The AUC for novel calcium scoring system is significantly larger $(0.925$ vs $0.706, p=0.002)$. 

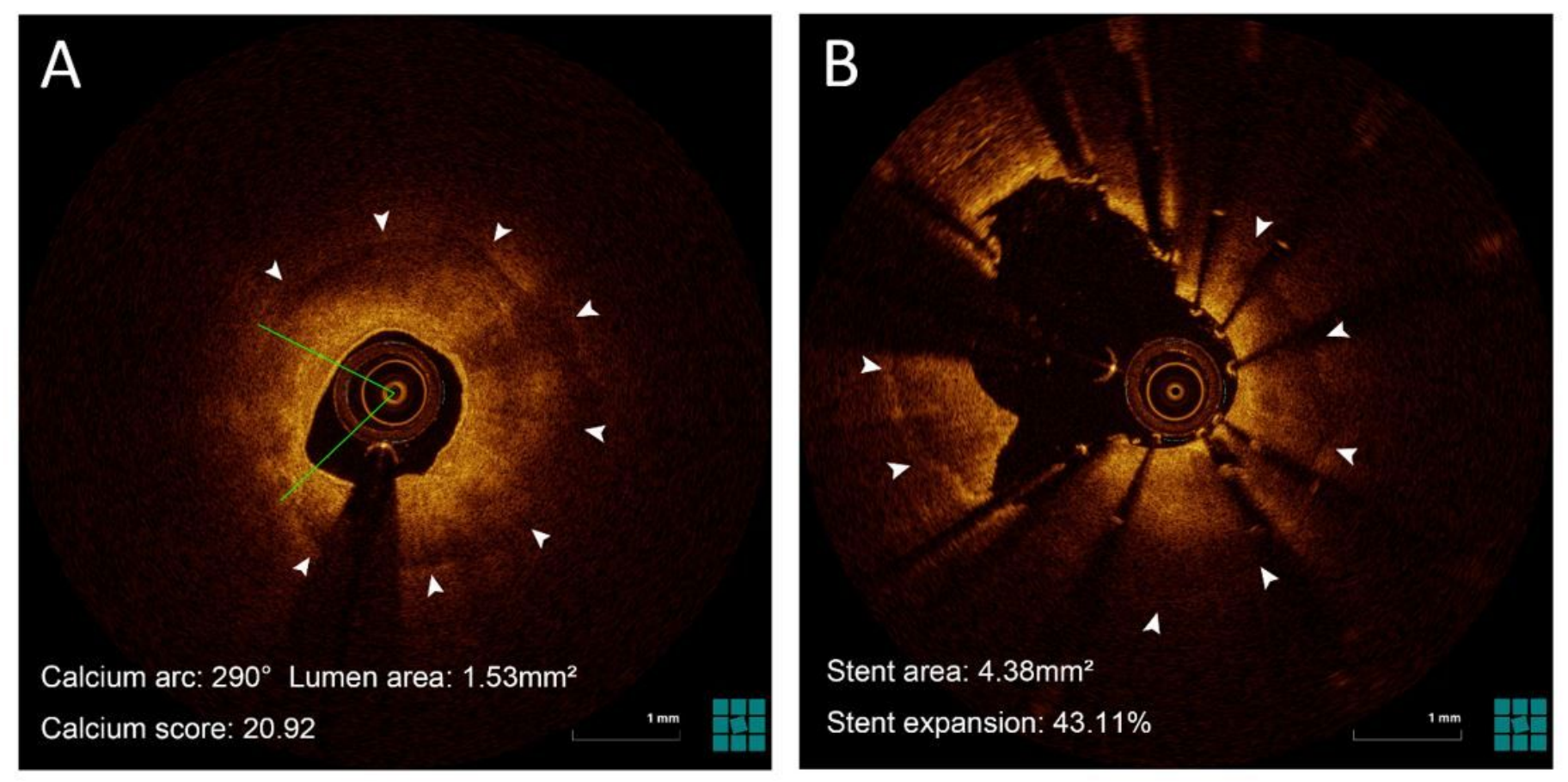

\section{Figure 3}

Representative optical coherence tomography (OCT) images. (A) The lesion manifested as severe calcified stenosis which had a calcium arc of $290^{\circ}$ (arrowhead), and minimum lumen area of $1.53 \mathrm{~mm}^{2}$ on pre-stent OCT image. The calculated calcium score of the lesion was 20.92. (B) The final OCT image after stent implantation showed poor stent expansion with minimum stent area only $4.38 \mathrm{~mm}^{2}$. 